\title{
A multivariate auto-regressive method to estimate cortico-muscular coherence for the detection of movement intent
}

\author{
G. Severini*, S. Conforto, M. Schmid and T. D’Alessio \\ Department of Applied Electronics, Roma TRE University, Rome, Italy
}

\begin{abstract}
In this work a time-frequency approach to estimate the Cortico-Muscular Coherence for the detection of the movement intent is presented, assessed on simulated data, and evaluated experimentally during different motor tasks performed by healthy subjects and patients suffering from different types of tremor. Cortico-Muscular Coherence is an index of the coupling of EEG signal in the cortical area with sEMG activity in the frequency domain, and its contributions in the beta band $(15-30 \mathrm{~Hz})$ have been associated to the movement intent. Cortico-Muscular Coherence estimation is here achieved by considering a closed-loop representation of the signals under analysis obtained through Multivariate Auto Regressive modeling. Significance levels for Cortico-Muscular Coherence are assessed by means of a surrogate data analysis approach. The detection technique is able to reveal significant Cortico-Muscular Coherence changes in $79 \%$ of the experimental trials, with a mean anticipation of 1.35 $\mathrm{s}$ with respect to movement onset. Time-frequency estimation of Cortico-Muscular Coherence can provide an insight for the development of a multimodal BCI able to integrate information from the brain activity in the functioning of assistive devices.
\end{abstract}

Keywords: Cortico-muscular coherence, movement intent, multimodal BCI, tremor control

\section{Introduction}

In the last years, many efforts have been dedicated to the study of how the motor cortex controls and synchronizes muscle activity during voluntary movements in humans. Some of these studies are based on the crossanalysis of cortical and muscular signals acquired simultaneously during various movement tasks.

Spectral coherence is an index of the coupling of two signals in the frequency domain and can be used to assess the common drives present in contemporary recordings of the two signals under analysis. If those

\footnotetext{
*Corresponding author: Giacomo Severini, Department of Applied Electronics, Roma; TRE University, Via della Vasca Navale 84, Roma 00146, Italy. Tel.: +390657337298; Fax: +390657337026; E-mail: gseverini@uniroma3.it.
}

signals are coming from cortical areas and muscular fibers, the term used is Cortico-Muscular Coherence (CMC) [1]. From a physiological point of view, CMC reflects the synchronization of motor units discharge at different frequencies by several descending drives.

Magnetoencephalographic recordings (MEG) in man show $\mathrm{CMC}$ between the controlateral cortical signal from the primary motor cortex and EMG activity in the beta band $(15-30 \mathrm{~Hz})$ during weak contractions of forearm and hand muscles [2,3]. These results have been obtained also using EEG signals [4]. The oscillatory drives in the beta band have been associated to voluntary muscular activity during submaximal contractions [1, 4]. As presented in [5], results obtained in this sense suggest that the rhythmic drive to human muscles in the beta band may originate in the primary motor cortex and contributes to the overall $\mu$ 
rhythm $(8-12 \mathrm{~Hz})$ recorded from the motor cortex. This rhythm disappears during voluntary movements, although some rhythmic activity re-appears during isometric contractions [6]. Coherence contribution in the band between 5 and $12 \mathrm{~Hz}$ seems instead to be related to the central elements of physiological tremor [2] and pulsatile organization of movement [7], although no convincing evidence of motor cortex drive has been proven, and only occasionally sub-peaks at these frequencies have been seen in coherence spectra of the MEG signal from the motor cortex and EMG activity [3].

In order to estimate CMC, many methods have been developed in the past years: the standard approach to coherence estimation is represented by Welch's Fourier-based periodogram $[1,4]$, while also timefrequency approaches based on wavelet analysis have been used to investigate coherence evolution during arm or finger movements $[6,8]$. Autoregressive modeling of the signals $[9,10]$ for CMC estimation has also been studied.

Since these CMC contributions can be caused either by cortical commands to the muscles or by the afferent feedback from the contracting muscles [11], autoregressive multivariate methods for signal analysis have been applied in order to study the directional coherence contributions.

If the assessment of the time evolution of the CMC is used to timely drive an assistive technology appliance, a real-time CMC estimation is needed. In this case, the standard Fourier-based method shows limitations due to the use of an analysis window whose length represents a critical issue.

In order to overcome the limitations of the standard Welch's approach, in this work we would like to apply a method based on bivariate auto-regressive (BAR) modeling [12] for the estimation of time-frequency CMC. In this formulation, CMC can be calculated from the parameters of the BAR model through a closed-loop representation of their generation. Besides being an alternative and efficient way to investigate time-frequency CMC contributions, the BAR approach can be tested as a component of a multimodal Brain Computer Interface (BCI) able to gain information on voluntary activity from $\mathrm{CMC}$ occurrences in the beta band. BCI is intended as a specialized interface that, using particular features extracted from the electrical activity of the brain, allows a subject to communicate directly to a computer or a computer-controlled device [13]. In order to make the BCI systems sufficiently accurate, the patient needs to be trained on how to control the assistive device, by voluntarily modulating the brain activity related to particular tasks. A different approach consists of extracting the control signals for the assistive device directly from the spontaneous brain activity, thus avoiding the training effort of the patient $[14,15]$.

The proposed work has been developed in the framework of the TREMOR project (FP7 action ICT-2007.7.2 "Accessible and Inclusive ICT", grant number ICT-2007-224051). The goal of this European Project is to develop a Functional Electrical Stimulation (FES)-based device able to counteract tremor without affecting the normal execution of voluntary movements. The final system outlined in TREMOR, that can be classified as an assistive technology instance, is intended to incorporate a movement intent section based on data coming from EEG and EMG signals.

In this manuscript, CMC is proposed as a solution for the detection of movement intent in tremor-affected patients and healthy subjects.

\section{Materials and methods}

\subsection{Coherence estimation}

Spectral coherence represents the correlation between two signals in the frequency domain. Coherence can be expressed by using its squared form, that is defined as in (1):

$$
\left|\mathrm{R}_{12}(\omega)\right|^{2}=\left|\mathrm{P}_{12}(\omega)\right|^{2} / \mathrm{P}_{11}(\omega) \mathrm{P}_{22}(\omega)
$$

where $\mathrm{P}_{12}(\omega)$ is the cross spectral density of the two processes $\mathrm{x}_{1}(n)$ and $\mathrm{x}_{2}(n)$ and $\mathrm{P}_{11}(\omega)$ and $\mathrm{P}_{22}(\omega)$ are the corresponding auto-spectral densities.

The estimation of $\mathrm{R}_{12}(\omega)$ for time-limited real signals can be obtained by either non-parametric [16] or parametric approaches. In the following, the focus will be on parametric techniques for CMC estimation, thus labeling EEG signal samples as $\mathrm{x}_{1}(n)$, and EMG signal samples as $\mathrm{x}_{2}(n)$.

\subsection{Signal modeling}

CMC can be estimated by using a BAR model of EEG and EMG signals. It has been chosen in order to overcome the time-frequency resolution problems 
of the Fourier-based methods, and to benefit from a modeling approach in terms of understanding the interconnections between two signals during their generation process. In BAR models, the current sample of each signal depends on past inputs of the signal itself and past inputs of the other signal, plus a White Gaussian Noise (WGN) realization sample of a given variance, thus making the generation of the two processes strongly interconnected, as described in (2).

$$
\begin{aligned}
x_{1}(n)= & -\sum_{k=1}^{\mathrm{p}} \mathrm{a}_{11}(k) x_{1}(n-k)- \\
& \sum_{k=1}^{\mathrm{p}} \mathrm{a}_{12}(k) x_{2}(n-k)+u_{1}(n) \\
x_{2}(n)= & -\sum_{k=1}^{\mathrm{p}} \mathrm{a}_{21}(k) x_{1}(n-k)- \\
& \sum_{k=1}^{\mathrm{p}} \mathrm{a}_{22}(k) x_{2}(n-k)+u_{2}(n)
\end{aligned}
$$

where $\mathrm{a}_{\mathrm{ij}}$ represent the coefficients of the BAR model, $u_{1,2}(n)$ represent the WGN realizations used as inputs, and $p$ is the order of the BAR model, that for the sake of simplicity has been chosen as the same for all the AR blocks.

Each set of coefficients $\left(a_{i j}\right)$ can be represented in the z-domain by (3):

$$
\begin{aligned}
& A_{i i}(z)=-\sum_{k=1}^{p} \mathrm{a}_{\mathrm{ii}}(\mathrm{k}) z^{-k}, \\
& A_{i j}(z)=-\sum_{k=1}^{p} \mathrm{a}_{\mathrm{ij}}(\mathrm{k}) z^{-k}
\end{aligned}
$$

These functions are then used to calculate the squared coherence between the two processes by means of a closed-loop representation of the whole process as explained in [12].

\subsection{Closed-loop representation and coherence estimation}

The transfer functions of the closed loop are represented in Fig. 1, and can be characterized from the coefficients of the autoregressive model (as in equation 3). The closed-loop blocks can be obtained from:

$$
\begin{aligned}
& H_{i}(Z)=1 /\left(1-A_{i i}(Z)\right) \\
& G_{i j}(Z)=A_{i j}(Z) /\left(1-A_{i i}(Z)\right)
\end{aligned}
$$

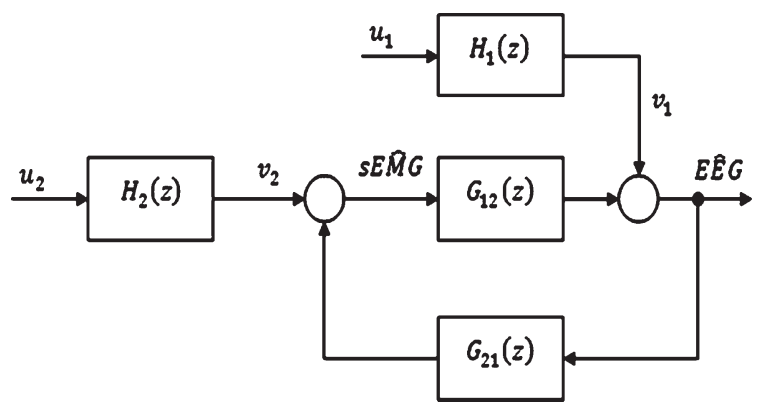

Fig. 1. Closed-loop representation of EEG and EMG signals generation.

From (5), the sensitivity function $S$ is defined as:

$$
S(Z)=\left(1-G_{12}(Z) G_{21}(Z)\right)^{-1}
$$

The auto-spectra and the cross-spectrum, and consequently the squared coherence in the frequency domain, can be obtained from the parameters just calculated as in equations 7,8 and 9 :

$$
\begin{aligned}
P_{11}(\omega)= & \left|S\left(e^{j \omega}\right) H_{11}\left(e^{j \omega}\right)\right|^{2} \gamma_{1}+\mid G_{12}\left(e^{j \omega}\right) \\
& \left.S\left(e^{j \omega}\right) H_{22}\left(e^{j \omega}\right)\right|^{2} \gamma_{2} \\
P_{22}(\omega)= & \left|S\left(e^{j \omega}\right) H_{22}\left(e^{j \omega}\right)\right|^{2} \gamma_{2}+\mid G_{21}\left(e^{j \omega}\right) \\
& \left.S\left(e^{j \omega}\right) H_{11}\left(e^{j \omega}\right)\right|^{2} \gamma_{1} \\
P_{12}(\omega)= & G_{21}\left(e^{-j \omega}\right)\left|S\left(e^{j \omega}\right) H_{11}\left(e^{j \omega}\right)\right|^{2} \gamma_{1}+ \\
& \left|G_{12}\left(e^{j \omega}\right) S\left(e^{j \omega}\right) H_{22}\left(e^{j \omega}\right)\right|^{2} \gamma_{2}
\end{aligned}
$$

where $\gamma_{1}$ and $\gamma_{2}$ are the covariance values of the two WGN realizations $u_{1}(n)$ and $u_{2}(n)$ feeding the closedloop. Following the definition of coherence, it is then possible to estimate the squared coherence as in (1).

This closed-loop representation of the EEG-EMG generation offers the possibility to estimate also the directional coherences, by alternatively zeroing the transfer functions of the blocks representing the crossdependencies of the signals (namely $\mathrm{G}_{12}$ and $\mathrm{G}_{21}$ ), thus splitting the contributions of spectral coherence into the two cause-effect directions.

\subsection{Time-frequency analysis}

In order to assess the time evolution of $\mathrm{CMC}$, the analysis has been implemented by segmenting the signals through an observation window sliding at fixed time steps. The window length and time shift are 
respectively $2.5 \mathrm{~s}$ and $0.06 \mathrm{~s}$. For each segment, the coefficients of a BAR model are estimated, and the coherence is correspondingly calculated, thus building a CMC time-frequency map. For the calculation of the AR parameters of the signals under examination, the package ARFIT [17, 18] has been used. The order of the bivariate AR process has been set at $p=13$.

\subsection{Significance level estimation}

Significance level estimation needs to be assessed in coherence estimation. In the Welch's approach, significance level is obtained according to [4], that depends on both the number of non-overlapping windows used to estimate the spectrum and the expected level of significance for the null hypothesis. In the case of AR spectral estimation, an alternative way of calculating the significance level is needed. An elegant solution in these regards is based on surrogate data analysis as presented in [19]: for each original signal pair, 40 surrogate pairs are calculated, randomly permuting the phase information of the Fourier transform of the series, thus destroying the original coherence coupling. Coherence is then obtained for each pair of the surrogate series, and, for each frequency value, the probability density function of the surrogate data values is estimated. The threshold is then set at the $100(1-\alpha)$ percentile, with $\alpha$ being the significance level chosen (in this case 0.05). The values of CMC that are below the threshold are considered as not significant and are thus zeroed in the final coherence map.

\subsection{Detection algorithm}

After assessing the significance level for the coherence map, a detection algorithm is performed in order to extract a digital signal where the level "high" indicates the presence of coherence contributions in the beta band. A threshold value equal to the mean value plus two times the standard deviation of the whole contribution of the coherence in the beta band in a given time step is calculated. For every time step, if the average of all the coherence values in the beta band exceeds the threshold, the control signal is set at the "high" level. If state transitions (both high and low) are shorter than $50 \mathrm{~ms}$, the algorithm does not take them into account.

\section{Performance on simulated data}

The first analysis performed on this method compares the time-frequency maps obtained by the BAR approach with those obtained by the Welch's periodogram on a set of simulated data. EEG signal has been simulated as a colored noise with frequency content between 0.5 and $100 \mathrm{~Hz}$, while EMG signal has been generated by modeling white noise through the Stulen-De Luca filter [22]. A common drive of narrowband $(4 \mathrm{~Hz})$ noise series has been added to both signals in the beta band in order to simulate CMC contributions for a fixed amount of time (11 s). Simulated time series have been processed in a time-frequency framework by using Welch's approach and the proposed BAR method. The length of the observation window and the time-step was the same for both analyses $(2.5 \mathrm{~s}$ and $0.06 \mathrm{~s}$, respectively). Both CMC maps underwent significance level estimation according to the method explained in section 2.5.

It can be noticed from Figs. $2 a$ and $2 b$ that the BAR approach offers a better frequency resolution as compared to the Welch's approach. In the BAR analysis the noise series common to both signals are recognizable, while in Welch's coherence analysis they cannot be distinguished.

\section{Performance on experimental data}

The method previously explained has then been used to analyze movements performed by two healthy subjects and four patients affected by rest tremor $(4-6 \mathrm{~Hz})$ and postural tremor $(5-8 \mathrm{~Hz})$, with different causing pathologies and different impairment levels.

\subsection{Experimental protocol}

Data have been recorded during three different motor tasks: a wrist task (WT), in which the subject is still in sitting position and performs a single wrist flexion-extension movement; an arms outstretched task (AOT), in which the subject is required to raise her/his arms and then maintain both the arms outstretched; a finger to nose task (FNT), in which the subject, starting from a rest position, has to move her/his finger to the nose 10 times. AOT and FNT tasks are preceded by a single wrist extension movement that has been used as a common reference between patients and tasks for the detection of actual movement intent. An acoustic cue 


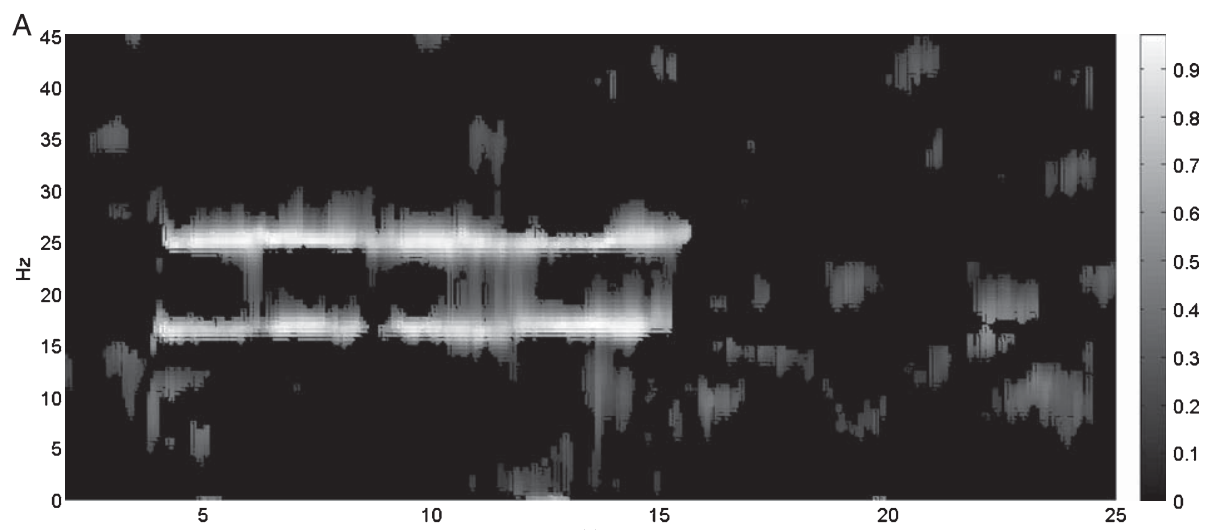

(s)

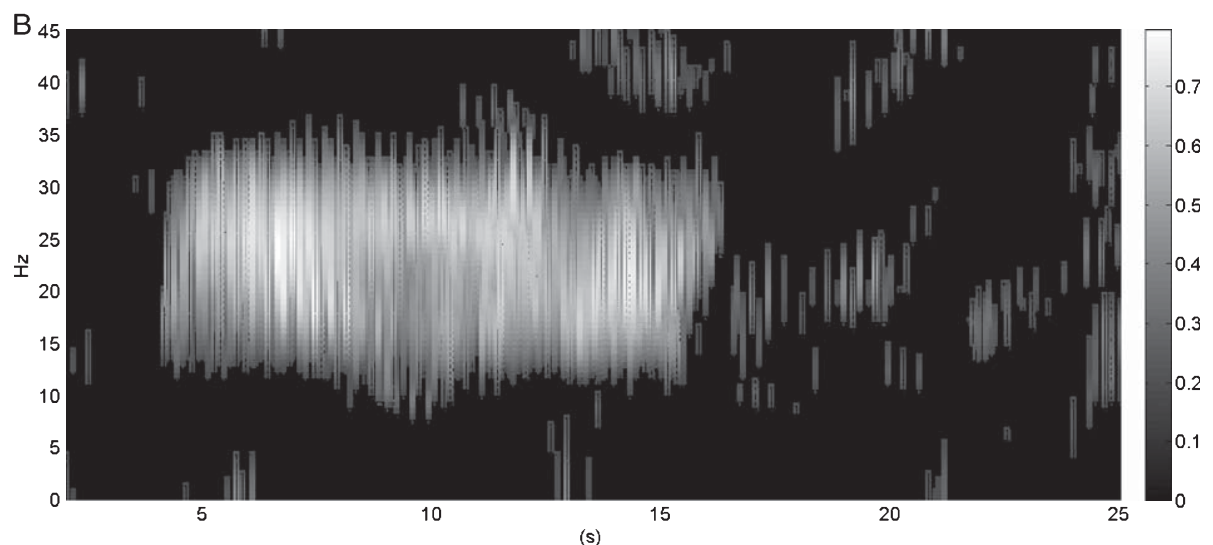

Fig. 2. Time-frequency coherence analysis performed using the proposed method (a) and the standard Welch's algorithm (b). Analysis has been performed on simulated EEG and EMG signals with two common narrowband (4 Hz) noise series, centered respectively around 17 and $23 \mathrm{~Hz}$, between 4 and 15 seconds.

was used to warn the subjects about the beginning of every task. All the recording sessions last between 30 and $50 \mathrm{~s}$.

EEG signals have been acquired at a sampling frequency of 1000 samples per second using tin electrodes; then they have been notch filtered at $50 \mathrm{~Hz}$ and low-pass filtered at $60 \mathrm{~Hz}$. The EMG signals have been recorded using two matrices of 6 electrodes [20] with an inter-electrode distance of $8 \mathrm{~mm}$. The best electrode (in terms of SNR) has been chosen and processed for each array. The muscles analyzed in this work, depending on the tasks previously indicated, are the Extensor Carpi Ulnaris (ECU), the Flexor Carpi Ulnaris (FCU) and the Biceps Brachii (BIC). The EMG signal has been notch filtered at $50 \mathrm{~Hz}$, rectified and then lowpass filtered at $60 \mathrm{~Hz}$ in order to maintain relevant CMC contributions in this band. Finally all the signals have been sub-sampled at a frequency of 150 samples per second. CMC has then been estimated by using EEG signals from $\mathrm{C} 3$ or $\mathrm{C} 4$ electrodes, (in a controlateral configuration with respect to the EMG) depending on the arm (right arm - C3; left arm - C4) and using EMG signals from the best electrode of the most relevant muscle involved in each task. A total of 33 trials have been analyzed.

In order to compare the timing of the coherence contributions in the beta band with the actual occurrence of the voluntary movement, an algorithm for the detection of muscular activation from the EMG signal has been used [21].

\subsection{Analysis on experimental data}

Figures 3 to 5 present three different examples of time-frequency CMC maps for the tasks taken into account, together with a rectified EMG plot for every map used as a timing reference for the actual movement. 


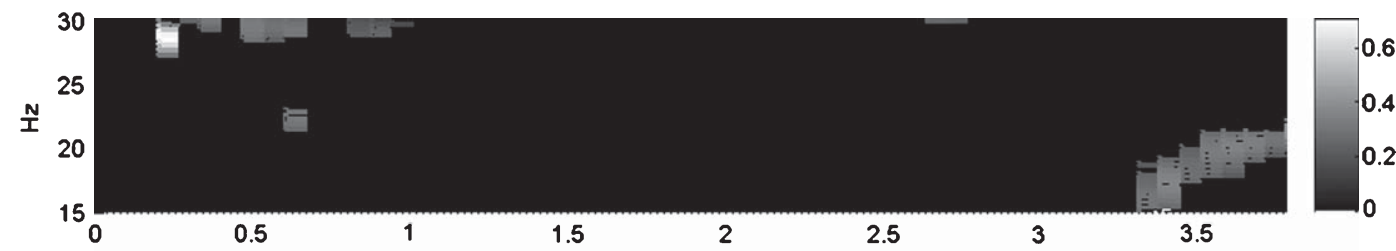

(s)

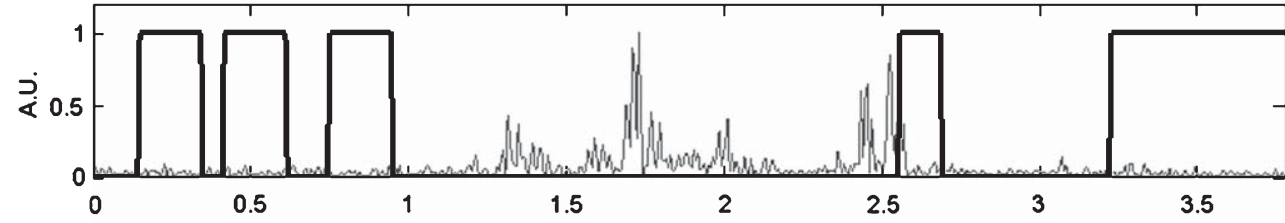

(s)

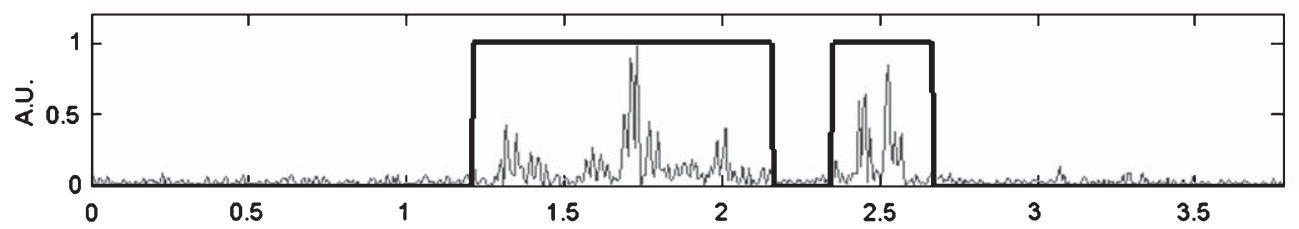

(s)

Fig. 3. Top panel represents coherence analysis between C3 electrode and EMG at the right ECU on a rest task for patient 01. Middle panel represents the digital output of the algorithm highlighting CMC contributions in the beta band. Bottom panel shows the corresponding EMG activity estimated using Bonato's algorithm.

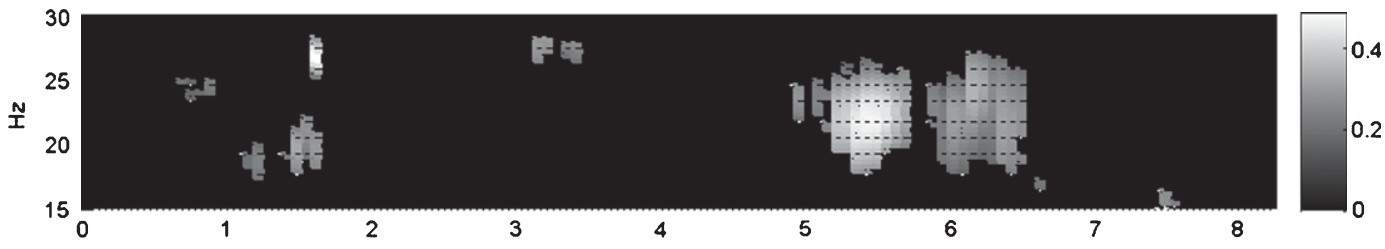

(s)

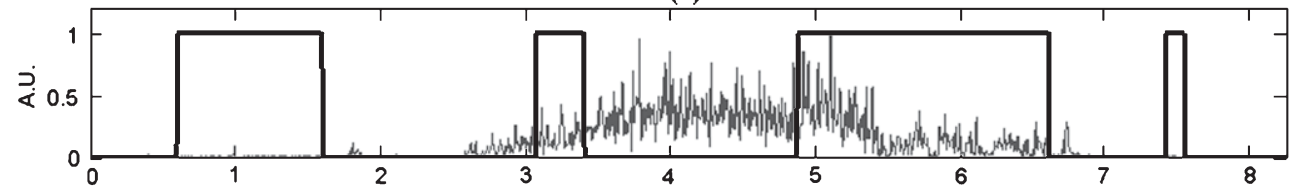

(s)

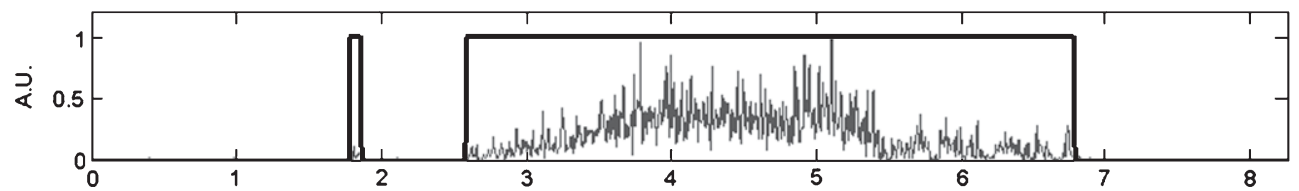

(s)

Fig. 4. Top panel represents coherence analysis between $\mathrm{C} 3$ electrode and EMG at the right ECU on a rest task for patient 02 . Middle panel represents the digital output of the algorithm highlighting CMC contributions in the beta band. Bottom panel shows the corresponding EMG activity estimated using Bonato's algorithm.

As previously shown, CMC contributions in the beta band have been associated to the planning and execution of the voluntary movement. So the aim of this analysis is to detect coherence intervals in this band before and during the actual movement, and to identify possible recurring patterns in patients and healthy 

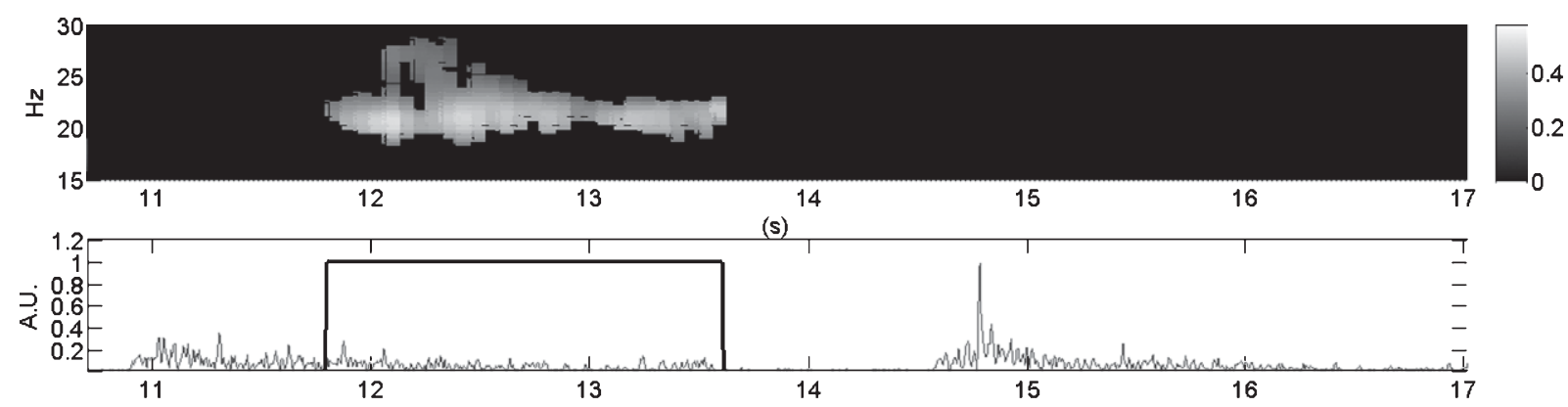

(s)

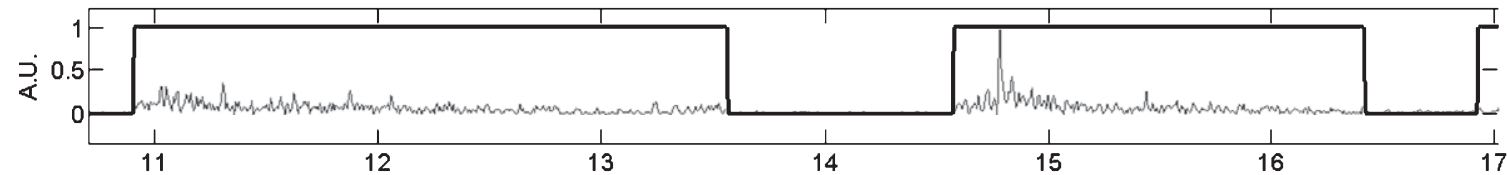

(s)

Fig. 5. Top panel represents coherence analysis between $\mathrm{C} 3$ electrode and EMG at the right BIC on a FN task for patient 04 during two consecutive movements. Middle panel represents the digital output of the algorithm highlighting CMC contributions in the beta band. Bottom panel shows the corresponding EMG activity estimated using Bonato's algorithm.

Table 1

Mean value, standard deviation and observed percentage on trials values for $\mathrm{CMC}$ contributions anticipating the execution of the first movement

\begin{tabular}{lccc}
\hline & $\begin{array}{c}\text { Mean } \\
\text { anticipation }\end{array}$ & $\begin{array}{c}\text { Standard } \\
\text { deviation }\end{array}$ & $\begin{array}{c}\text { Observed } \\
\text { percentage }\end{array}$ \\
\hline Patients & $1.41 \mathrm{~s}$ & $0.47 \mathrm{~s}$ & $77 \%$ \\
Controls & $1.06 \mathrm{~s}$ & $0.28 \mathrm{~s}$ & $83 \%$ \\
Overall & $1.35 \mathrm{~s}$ & $0.45 \mathrm{~s}$ & $79 \%$ \\
\hline
\end{tabular}

subjects. It can be noticed from the figures that distinctive significant coherence values can be detected in the beta band right before $(0.5 \mathrm{~s}$ to $2.5 \mathrm{~s})$ the beginning of the first (wrist) movement in the tasks taken into account.

This behaviour has been observed in $79 \%$ of the analyzed trials ( $83 \%$ in controls, $77 \%$ in patients) with a mean value of anticipation of $1.35 \mathrm{~s}$ with respect to the actual movement, and a standard deviation of $0.45 \mathrm{~s}$ (see Table 1).

Also in $50 \%$ of the analyzed trials no significant CMC contributions in the beta band have been observed during the execution of the first (wrist) movement (as in Fig. 3). As observed in the literature [1, 6], sustained movements present high beta-band coherence contributions during the execution (as shown in Fig. 4). Nevertheless, in tasks where several abrupt movements are present (Fig. 5), it is not possible to distinguish among CMC changes associated with each single movement.

\section{Discussion and conclusions}

In this work a method based on bivariate autoregressive (BAR) modelling for the estimation of time-frequency CMC has been applied and tested on simulated signals and on data recorded on two healthy subjects and four patients affected by tremor impairment. Results on simulated signals show that this method is able to provide better results in the time-frequency domain with respect to the Welch's approach. Analysis on data from experimental trials shows how coherence contributions in the beta-band, which have been linked in literature to the execution of voluntary movements, show significant values before and during the actual execution of the tasks. It can be speculated that $\mathrm{CMC}$ contributions preceding the beginning of the current movement could be caused by the descending drives modulating tonic muscular activity, triggered by the acoustic cue indicating the beginning of the task [23].

Nevertheless, it is not possible to gain stable information in abrupt movements. No particular differences have been noticed between healthy subjects and tremulous patients in the tasks taken into account, although it has to be pointed out that no major tremor episodes were present during these trials.

The algorithm proposed in this work is implementable in real-time, with the limitation of a buffer given by the presence of the analysis window. By prop- 
erly selecting the length of the observation window and the time step of the analysis, it is possible to overcome the computational complexity of the approach that is now limited by the procedure for significance level estimation. The BAR model is also able to provide, with respect to the Welch's approach, a better frequency resolution.

This work proves the validity of the use of a BAR approach in the estimation of CMC. The information obtained using this method can be integrated with information regarding muscular activations (related both to tremor and voluntary movement) and with that coming from kinematic and kinetic sensors. In particular, CMC contributions in the beta band prior to the execution of a given movement can be used as an index of the beginning of voluntary activity, thus putting the basis for the possible integration of this method in a multimodal BCI-driven control system, which takes into account joint contributions gained from both EEG and EMG signals.

\section{Acknowledgments}

The research work presented in this paper has been carried out with the financial support of the European Union Commission, within FP7 action ICT-2007.7.2 "Accessible and Inclusive ICT", under contract number grant number ICT-2007-224051 TREMOR “An ambulatory BCI-driven tremor suppression system based on functional electrical stimulation". The authors would like to thank the partners of the TREMOR Project consortium for the support and the joint work in the common experimental session organized in Bruxelles in January 2009.

\section{References}

[1] B.A. Conway, D.M. Halliday, S.F. Farmer, U. Shahani, P. Maas, A.I. Weir and J.R. Rosenberg, Synchronization between motor cortex and spinal motoneuronal pool during the performance of a maintained motor task in man, The Journal of Physiology 489 (Pt 3) (1995), 917-924.

[2] B.A. Conway, S.F. Farmer, D.M. Halliday and J.R. Rosenberg, On the relation between motor-unit discharge and physiological tremor. Alpha and Gamma Motor Systems, New York (USA), Plenum Press, (1995), 596-598.

[3] S. Salenius, K. Portin, M. Kajola, R. Salmelin and R. Hari, Cortical control of human motoneuron firing during isometric contraction, Journal of Neurophysiology 77 (1997), 3401-3405.

[4] D.M. Halliday, B.A. Conway, S.F. Farmer and J.R. Rosenberg, Using electroencephalography to study functional coupling between cortical activity and electromyograms during voluntary contractions in humans, Neuroscience Letters 241 (1998), 5-8.

[5] P. Brown, Cortical drives to human muscle: the Piper and related rhythms, Progress in Neurobiology 60 (2000), 97-108.

[6] J.M. Kilner, S.N. Baker, S. Salenius, R. Hari and R.N. Lemon, Human cortical muscle coherence is directly related to specific motor parameters, The Journal of neuroscience: the Official Journal of the Society for Neuroscience 20 (2000), 8838-8845.

[7] A.B. Vallbo and J. Wessberg, Organization of motor output in slow finger movements in man, The Journal of Physiology 469 (1993), 673-679.

[8] Y. Zhan, D. Halliday, P. Jiang, X. Liu and J. Feng, Detecting time-dependent coherence between non-stationary electrophysiological signals-a combined statistical and timefrequency approach, Journal of Neuroscience Methods 156 (2006), 322-332

[9] F. Panzica, L. Canafoglia, S. Franceschetti, S. Binelli, C. Ciano, E. Visani and G. Avanzini, Movement-activated myoclonus in genetically defined progressive myoclonic epilepsies: EEG-EMG relationship estimated using autoregressive models, Clinical Neurophysiology: Official Journal of the International Federation of Clinical Neurophysiology 114 (2003), 1041-1052.

[10] H. Kato and M. Honda, Multiplicatively modulated exponential autoregressive model for corticomuscular functional coupling. Signal Processing 85 (2005), 1287-1300.

[11] T. Mima, K. Toma, B. Koshy and M. Hallett, Coherence between cortical and muscular activities after subcortical stroke, Stroke; a Journal of Cerebral Circulation 32 (2001), 2597-2601.

[12] G. Baselli, E. Caiani, A. Porta, N. Montano, M.G. Signorini and S. Cerutti, Biomedical signal processing and modeling in cardiovascular systems, Critical Reviews in Biomedical Engineering 30 (2002), 55-84.

[13] T.M. Vaughan, W.J. Heetderks, L.J. Trejo, W.Z. Rymer, M. Weinrich, M.M. Moore, A. Kubler, B.H. Dobkin, N. Birbaumer, E. Donchin, E.W. Wolpaw and J.R. Wolpaw, Brain-computer interface technology: a review of the Second International Meeting, IEEE transactions on neural systems and rehabilitation engineering: a publication of the IEEE Engineering in Medicine and Biology Society 11 (2003), 94-109.

[14] O. Bai, P. Lin, S. Vorbach, M.K. Floeter, N. Hattori and M. Hallett, A high performance sensorimotor beta rhythmbased brain-computer interface associated with human natural motor behavior, Journal of Neural Engineering 5 (2008), 24-35.

[15] N.J. Hill, T.N. Lal, M. Schroder, T. Hinterberger, B. Wilhelm, F. Nijboer, U. Mochty, G. Widman, C. Elger, B. Scholkopf, A. Kubler, N. Birbaumer, Classifying EEG and ECoG signals without subject training for fast BCI implementation: comparison of nonparalyzed and completely paralyzed subjects, IEEE Transactions on Neural Systems and Rehabilitation Engineering: A publication of the IEEE Engineering in Medicine and Biology Society 14 (2006), 183186.

[16] P.D. Welch, The Use of Fast Fourier Transform for the Estimation of Power Spectra: A Method Based on Time Averaging Over Short, Modified Periodograms, IEEE Trans Audio Electroacoustics 15 (1967), 70-73. 
[17] A. Neumaier and T. Schneider, Estimation of parameters and eigenmodes of multivariate autoregressive models, $A C M$ Trans Math Softw 27 (2001), 27-57.

[18] T. Schneider and A. Neumaier, Algorithm 808: ARfit - A Matlab package for the estimation of parameters and eigenmodes of multivariate autoregressive models, ACM Trans Math Softw 27 (2001), 58-65.

[19] L. Faes, A. Porta, R. Cucino, S. Cerutti, R. Antolini and G. Nollo, Causal transfer function analysis to describe closed loop interactions between cardiovascular and cardiorespiratory variability signals, Biological Cybernetics 90 (2004), 390-399.

[20] D. Farina, W. Muhammad, E. Fortunato, O. Meste, R. Merletti and H. Rix, Estimation of single motor unit conduction velocity from surface electromyogram signals detected with linear electrode arrays, Medical \& Biological Engineering \& Computing 39 (2001), 225-236.
[21] P. Bonato, T. D'Alessio and M. Knaflitz, A statistical method for the measurement of muscle activation intervals from surface myoelectric signal during gait, IEEE Transactions on Biomedical Engineering 45 (1998), 287-299.

[22] F.B. Stulen and C.J. DeLuca, Frequency parameters of the myoelectric signal as a measure of muscle conduction velocity, IEEE Transactions on Biomedical Engineering 28 (1981), 515-523.

[23] A.G. Androulidakis, L.M. Doyle, K. Yarrow, V. Litvak, T.P. Gilbertson and P. Brown, Anticipatory changes in beta synchrony in the human corticospinal system and associated improvements in task performance, The European Journal of Neuroscience 25 (2007), 3758-3765. 

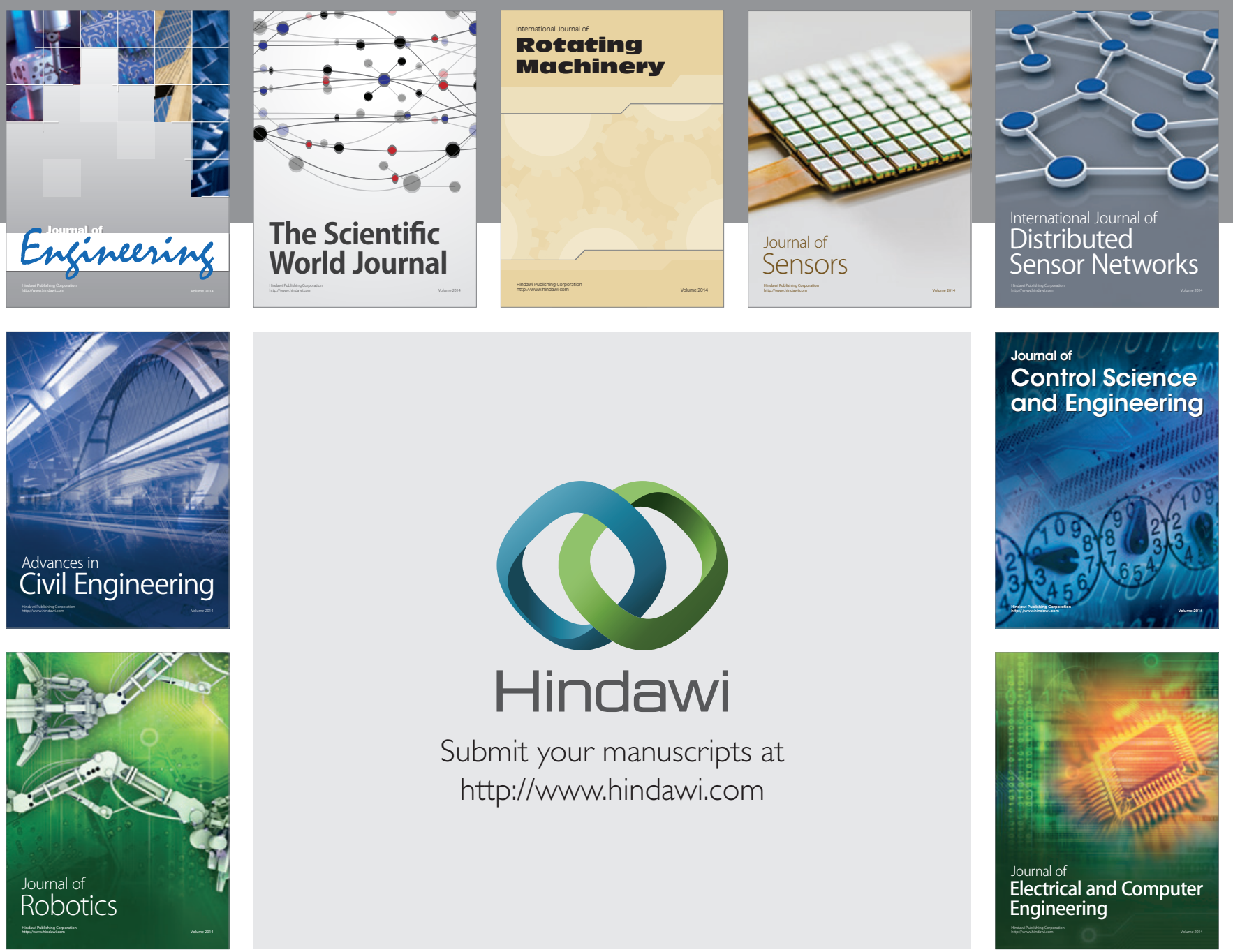

Submit your manuscripts at

http://www.hindawi.com
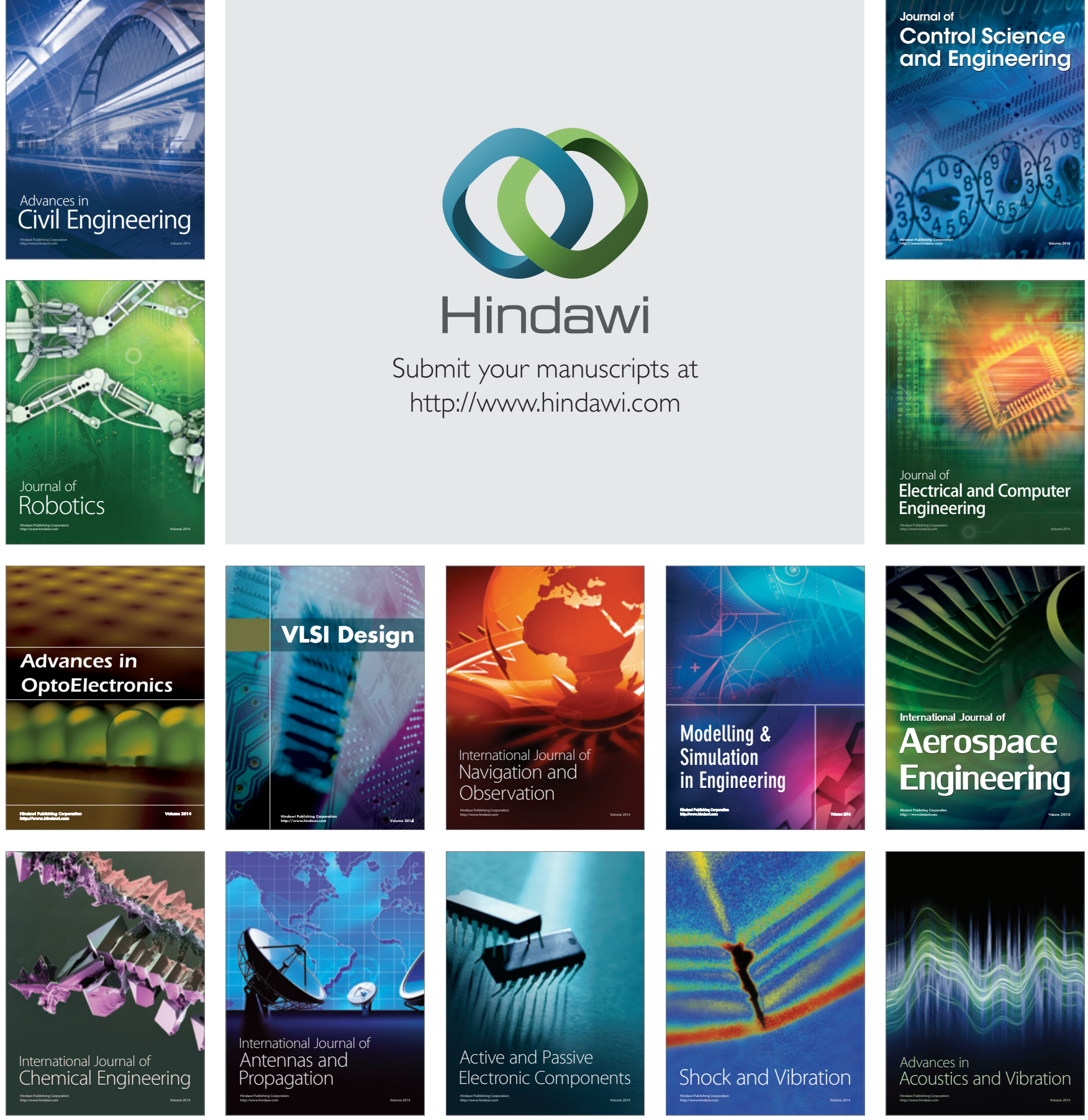